Corrigendum

\title{
Corrigendum to "Modular Self-Reconfigurable Robotic Systems: A Survey on Hardware Architectures"
}

\section{S. Sankhar Reddy Chennareddy, Anita Agrawal, and Anupama Karuppiah}

EEE Department, NH-17B, BITS Pilani KK Birla Goa Campus, Goa 403726, India

Correspondence should be addressed to S. Sankhar Reddy Chennareddy; sankhar@goa.bits-pilani.ac.in

Received 3 July 2017; Accepted 26 July 2017; Published 21 August 2017

Copyright (C) 2017 S. Sankhar Reddy Chennareddy et al. This is an open access article distributed under the Creative Commons Attribution License, which permits unrestricted use, distribution, and reproduction in any medium, provided the original work is properly cited.

In the article titled "Modular Self-Reconfigurable Robotic Systems: A Survey on Hardware Architectures" [1], the data presented in Table 2 might have been confusing. Therefore, the table is reformatted as follows. 


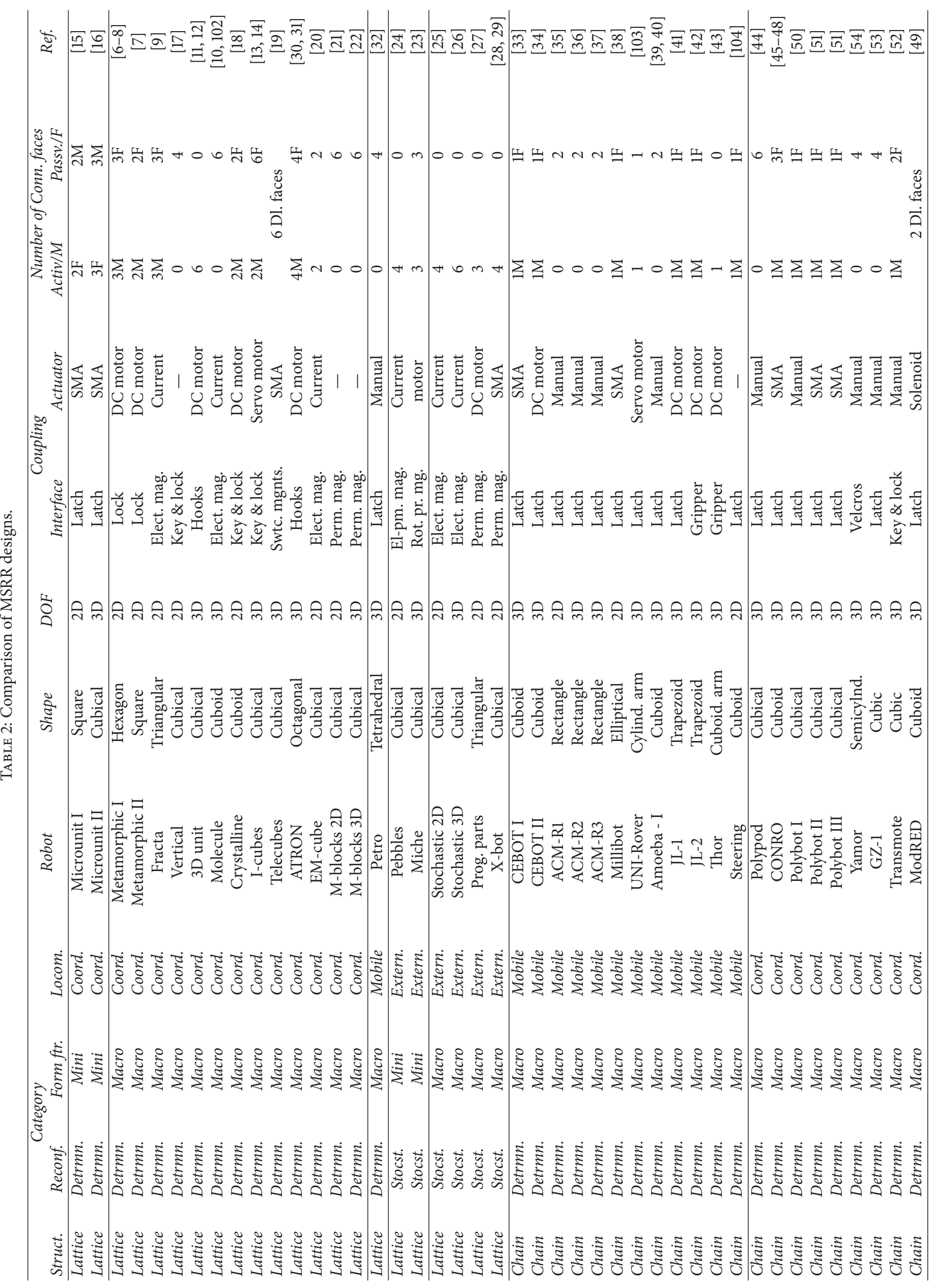




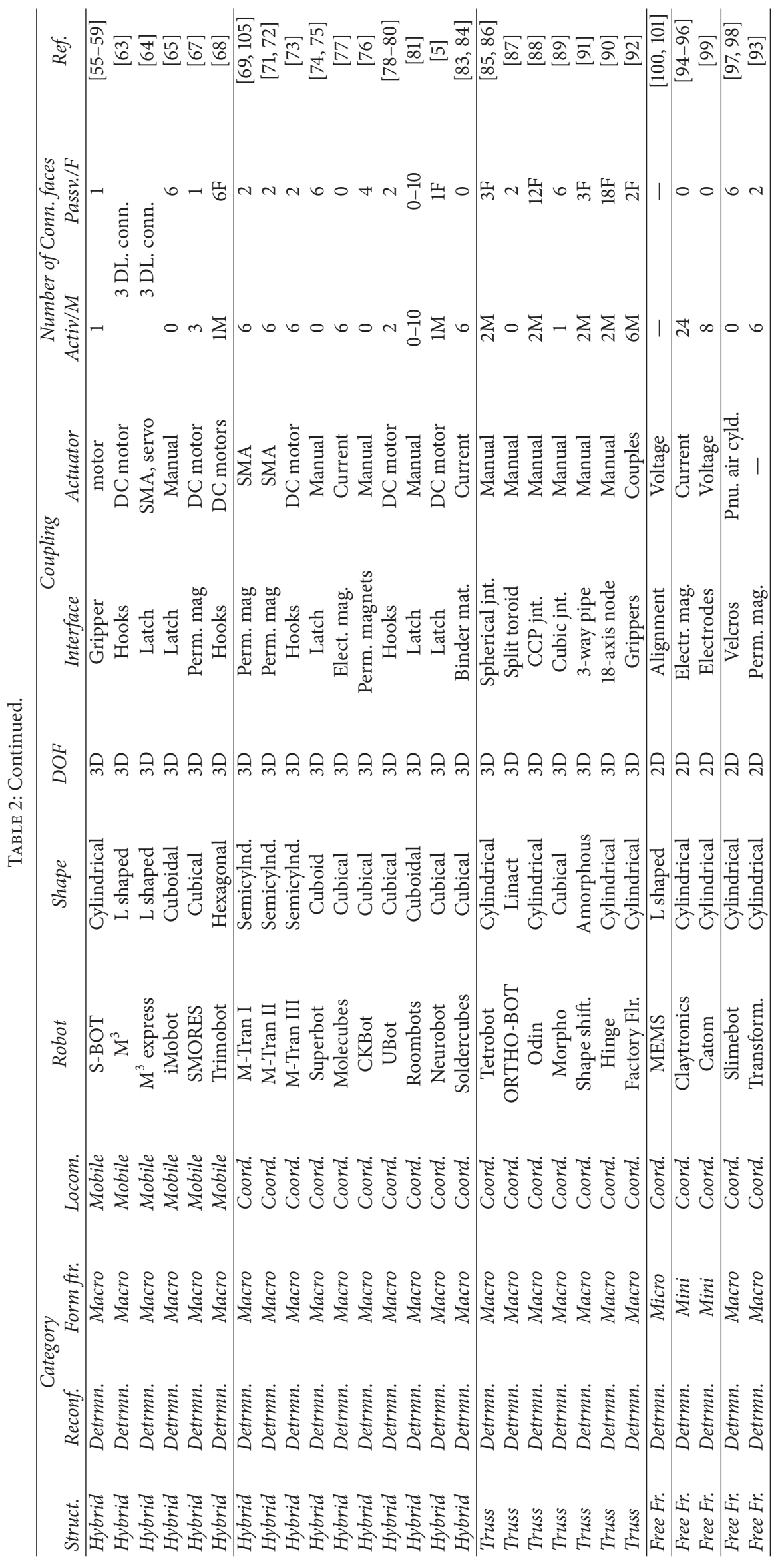




\section{References}

[1] S. S. R. Chennareddy, A. Agrawal, and A. Karuppiah, "Modular self-reconfigurable robotic systems: a survey on hardware architectures," Journal of Robotics, vol. 2017, Article ID 5013532, 19 pages, 2017. 


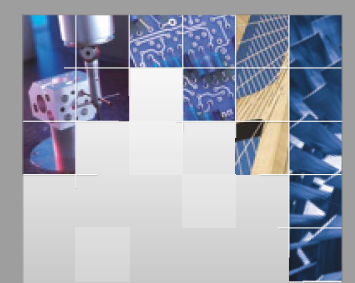

\section{Enfincering}
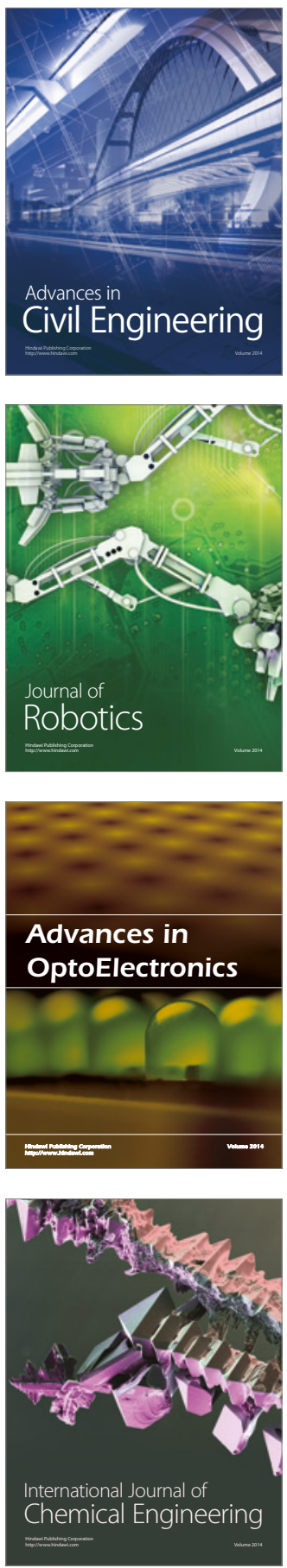

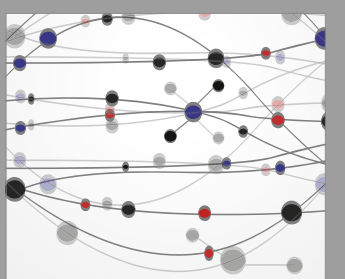

The Scientific World Journal

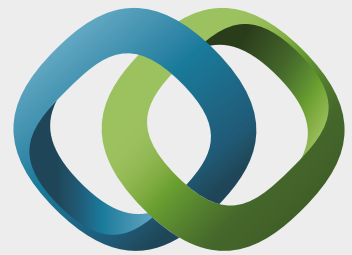

\section{Hindawi}

Submit your manuscripts at

https://www.hindawi.com
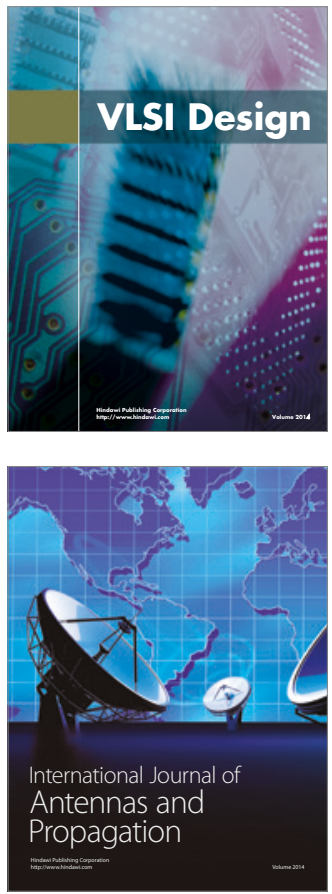

\section{Rotating}

Machinery
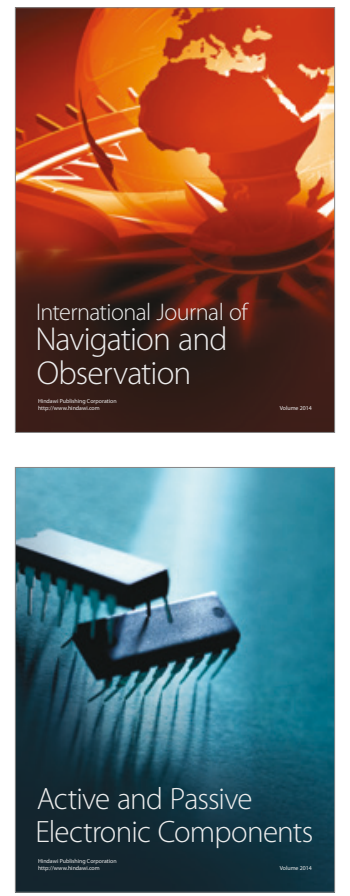
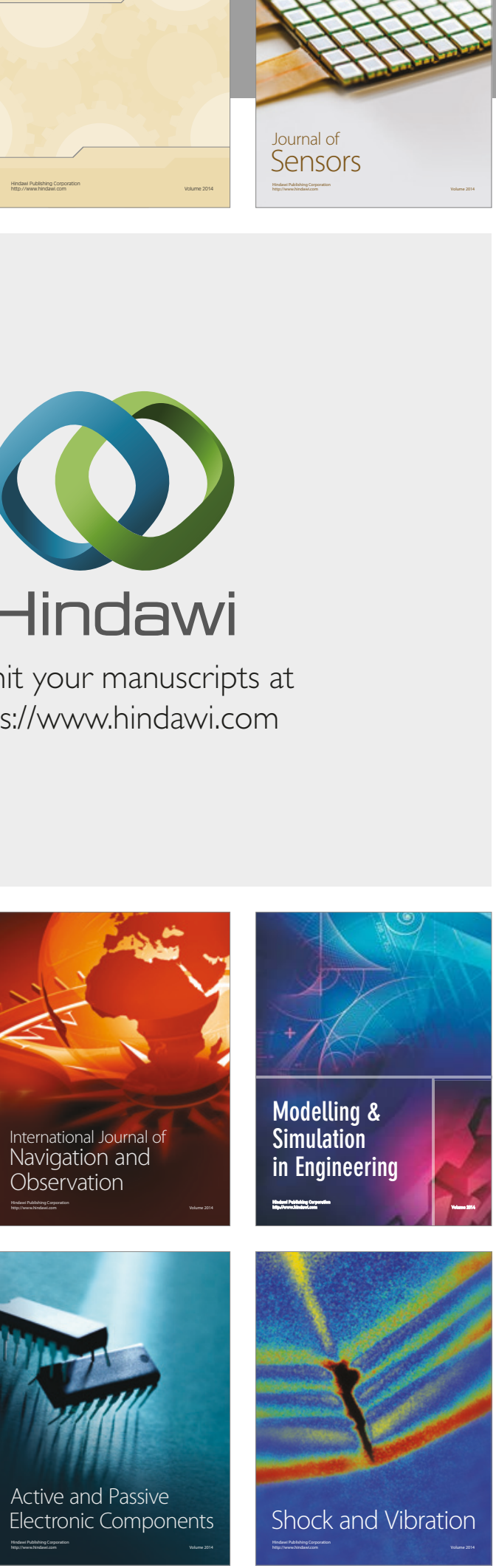
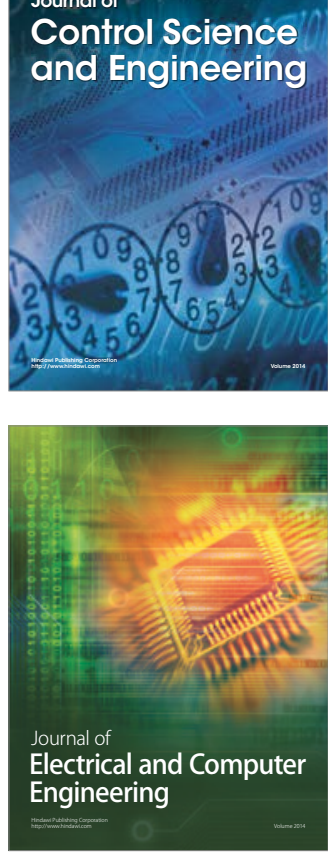

Distributed

Journal of

Control Science

and Engineering
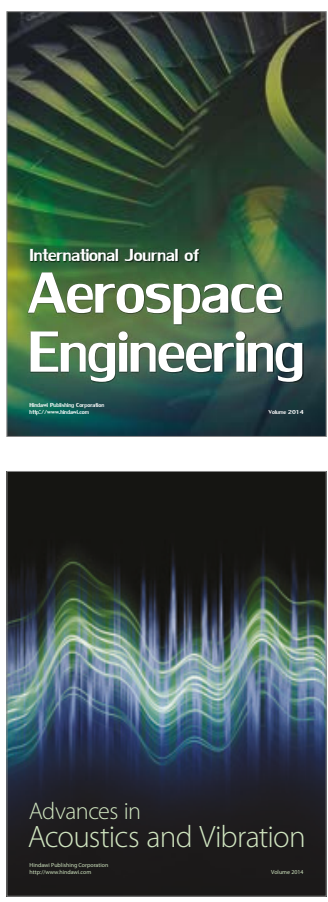

Sensor Networks 